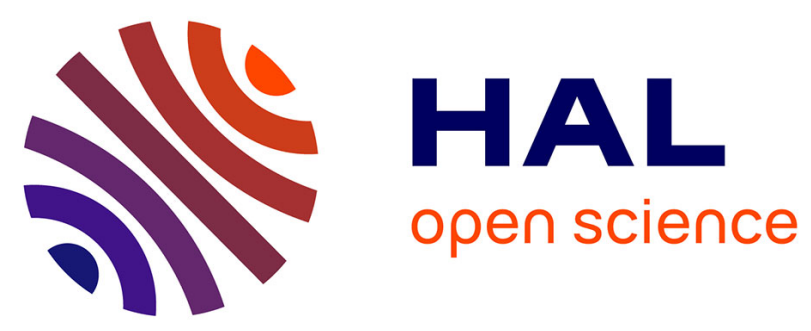

\title{
Photobleaching as a tool to measure the local strain field in fibrous membranes of connective tissues
}

\author{
Charles Jayyosi, Guillaume Fargier, Michel Coret, Karine Bruyere-Garnier
}

\section{To cite this version:}

Charles Jayyosi, Guillaume Fargier, Michel Coret, Karine Bruyere-Garnier. Photobleaching as a tool to measure the local strain field in fibrous membranes of connective tissues. Acta Biomaterialia, 2014, 10 (6), pp.2591-2601. 10.1016/j.actbio.2014.02.031 . hal-01546501v2

\section{HAL Id: hal-01546501 \\ https://hal.science/hal-01546501v2}

Submitted on 19 Feb 2018

HAL is a multi-disciplinary open access archive for the deposit and dissemination of scientific research documents, whether they are published or not. The documents may come from teaching and research institutions in France or abroad, or from public or private research centers.
L'archive ouverte pluridisciplinaire HAL, est destinée au dépôt et à la diffusion de documents scientifiques de niveau recherche, publiés ou non, émanant des établissements d'enseignement et de recherche français ou étrangers, des laboratoires publics ou privés. 
FARGIER, Charles, CORET, Guillaume, BRUYERE-GARNIER, Michel, 2014, Photobleaching as a tool to measure the local strain field in fibrous membranes of connective tissues, Acta Biomaterialia, 10, 6, ELSEVIER, pp. 2591-2601, DOI: 10.1016/j.actbio.2014.02.031

\title{
Photobleaching as tool to measure local strain field in fibrous membranes of connective tissues
}

\author{
C. Jayyosi*1, G. Fargier ${ }^{2}$, M. Coret ${ }^{3}$ and K. Bruyère-Garnier ${ }^{1}$ \\ ${ }^{1}$ Université de Lyon, F-69622 Lyon ; IFSTTAR, LBMC, UMR-T9406 ; Université Lyonl, France \\ ${ }^{2}$ Plateforme IVTV, CNRS, 36 Avenue Guy de Collongue Bâtiment G8 69134 Ecully Cedex, France \\ ${ }^{3}$ LUNAM Université, GEM, UMR CNRS 6183, Ecole Centrale de Nantes, Université de Nantes, France
}

Keywords: photobleaching; strain measurement; liver's capsule; collagen; elastin; two-photon excited fluorescence; second harmonic generation

\begin{abstract}
Connective tissues are complex structures which contain collagen and elastin fibers. These fiber based structures have a great influence on material mechanical properties and need to be studied at the microscopic scale. Several microscopy techniques have been developed in order to image such microstructures; among them are two-photon excited fluorescence microscopy and second harmonic generation. These observations have been coupled with mechanical characterization to link microstructure kinematic to macroscopic material parameter evolution. In this study, we present a new approach to measure local strain in soft biological tissues using a side effect of fluorescence microscopy: photobleaching. Controlling the loss of fluorescence induced by photobleaching, we create a pattern on our sample that we can monitor during mechanical loading. The image analysis allows computing 3D displacements of the patterns at various loading levels. Then, local strain distribution is derived using the finite element discretization on a four nodes element mesh created from our photobleached pattern. Photobleaching tests on human liver's capsule have revealed that this technique is non-destructive and has not any impact on mechanical properties. This method is likely to have other applications in biological material studies, considering that all collagen-elastin fibers based biological tissues possess autofluorescence properties, and thus can be photobleached.
\end{abstract}




\section{Introduction}

Study of soft biological tissues behavior has an important position in biomechanics interests, mainly because of its use for modeling purposes in various fields such as road safety or biomedical applications for instance. At the macro scale, many mechanical tests have been put in place to assess biological material properties. For fibrous connective membranes, different experiments allowed to investigate their global behavior, calculating failure stresses, apparent modulus, global strain [1]-[5].

However, there is a much more limited understanding of connective tissues micromechanics and how the fiber composite structure reacts to loading and provides such mechanical behaviors. Few studies, mostly about tendons, were conducted in order to investigate the changes in the microstructure during mechanical loading. They address fundamental questions about material characterization and gives precious details about cellular strain environment. Screen et al. conducted a series of experiments in which they monitored the displacement of cell nuclei in tendons submitted to uniaxial tensile test, to compute local strain of collagen fibers and understand the tendon extension mechanism [6]-[8]. Sasaki et al. [9] also worked on explaining tendons extension linking it to different behaviors of collagen fibers at different scales, up to the molecular one. More recently, Houssen et al. [10] performed uniaxial tensile test on rat tail tendon and observed microstructure re-organization during loading, using Second Harmonic Generation (SHG) signal from collagen fibers. Keyes et al. investigated vascular tissues behavior from a microstructural point of view developing a pressure loading device and using multiphoton microscopy [11], [12]. Such studies provide a brand new insight into natural material design and allow understanding on how fiber composite structures influence the macroscopic behavior.

In order to perform such investigation of soft biological tissue microstructures, many imagery techniques have been used. Among them, we find X-ray diffraction [9], [13], [14], polarized light [15], [16], SHG[10], confocal microscopy [6], [7], [17], [18], and a combination of SHG and two-photon excited fluorescence (TPEF) with multiphoton microscopy [11], [12], [19]-[22].

Fluorescence microscopy is now well established as a valuable tool to observe soft biological tissues microstructure. It has been widely used on many connective tissues, since their main components, which are collagen and elastin, have autofluorescence properties. Indeed, those two kinds of fibers each have several fluorophores [23]-[25], which allow their direct observation when excited at a specific wavelength, without requiring use of specific fluorescent markers. Unfortunately, emission spectra of collagen and elastin overlap significantly, preventing them to be distinguished after excitation at a commune wavelength [26].

On the other hand, collagen has a molecular organization that generates second harmonics [10], [21], [27]. Therefore it can be observed with multiphoton microscopy at precisely half the excitation wavelength. Considering those properties, it is thus possible to observe collagen and elastin fibers while making the distinction between them, by parting two-photon excited fluorescence (TPEF) signal and SHG signal, which will show elastin and collagen respectively. Some studies already used those techniques to image collagen and elastin fibers simultaneously using only one two-photon excitation microscope [11], [21], [22].

One of the usual drawbacks of fluorescence microscopy is photobleaching. This phenomenon induces a loss of fluorophores' fluorescence which implies a decrease of signal/noise ratio (SNR) and thus, reduces the quality of the images [28], [29]. Briefly, during the excitation of the fluorophore, molecules undergo permanent structural changes by reacting and creating covalent bonds with other species, especially oxygen. These changes often lead to an irreversible loss of fluorescence [30], [31]. Photobleaching mechanisms are still not perfectly known nowadays, however, many studies focused on the factors enhancing this usually disturbing phenomenon. Among those with great influence that have been reported, we notice the specific fluorophore considered, exciting photon flow intensity, irradiation duration, oxygen concentration and more globally, the environment nature [30], [32]-[36].

However, some microscopy techniques take advantage of that fluorescent loss to their benefit, like FRAP (Fluorescence Recovery After Photobleaching) which allows measuring flow parameters such as diffusion coefficient or molecule's speed among others [37], [38]. FLAP (Fluorescence Localization After Photobleaching) is another technique developed by Dunn et al. [39], which uses photobleaching to tag and track molecules. Cheng and Screen [40] proposed a use of photobleaching via classic confocal microscopy, to assess local strain in tendons after staining with fluorescent markers. They created lines of photobleaching resulting in a grid on which they measured several parameters to characterize the local strain.

There are several differences between photobleaching induced by one or multi-photon excitation. Indeed, considering the fact that multi-photon excitation requires a much more powerful intensity excitation than onephoton excitation, photobleaching is stronger in TPEF [31], [41]. With TPEF, photobleaching is also more 
localized, due to the confinement of excitation in a limited focal volume. Thus, TPEF provides limited photodamage within the sample and allows a more accurate control of the photobleached area borders [31].

Overall, photobleaching is a rather poorly understood phenomenon, and to our knowledge, there has not been any study which investigated its impact on biological tissues mechanical properties.

In view of modeling human abdominal organs, we focus on the study of liver's capsule mechanical behavior. As mentioned previously [11], [12], [20], in order to study the contribution of microstructure to fibrous connective tissues mechanical behavior at the macro scale, standard mechanical loading associated to observation of fibers by TPEF and SHG offers a great potential. Moreover, the strain field at the microscopic scale is important to assess in such mechanical analysis, and the use of TPEF and SHG to this end is rarely reported. Only Screen and Evans applied a FE modeling approach using cells as markers to compute a strain field [8].

Using the same approach as Screen and Evans [8], we propose the use of photobleaching to create markers for a strain field measurement. The aim of this paper is first to demonstrate that this technique is non-destructive and does not affect a priori mechanical properties, and secondly to show the feasibility of the method to measure local strain field.

\section{Materials and methods}

\section{Samples preparation}

Human liver's capsules were removed from a liver of post mortem human subject from the Department of Anatomy of the University of Rockefeller (DUAR, Lyon, France) through the French voluntary corpse donation to Science program. Capsule was delicately taken off using a syringe and water to create an hematoma, and then cut into dog bone shaped samples using a die cutter, giving a $45 \mathrm{~mm}$ length and a $6 \mathrm{~mm}$ section. Figure 1a) shows one of our samples' geometry. The dog bone shape was initially chosen to investigate rupture behavior which is not presented in this paper. Samples $(n=4)$ were stored at $4^{\circ} \mathrm{C}$ until testing, which was performed within 2 days after preparation, so that the samples were never frozen. They were kept hydrated in saline solution until testing.

\section{Microstructural observation}

A two-photon excitation microscope (NIKON, A1R MP PLUS®) was used to observe the liver's capsule microstructure. The excitation wavelenght was set to $850 \mathrm{~nm}$, which is deliberatly slightly above the values reported in the litterature $-780 \mathrm{~nm}$ in Keyes et al. [11], 800nm in Zoumi et al. [22] - to avoid collagene autofluorescence by exciting outside its excitation spectra while excitating elastin slightly hight in its spectra. Second harmonic generated light from collagen and autofluorescent light from elastin were collected through two chanels using respectively 2 filters: 400-492nm and 500-550nm. Image field of view was 507 x $507 \mu \mathrm{m}^{2}$ with a scan speed of 2 s/image and a resolution of $0.99 \mu \mathrm{m} /$ pix. To observe the changes along the entire thickness of the capsule, we imaged stacks of approximatively 60-80 $\mu \mathrm{m}$ depending of the capsule thickness, with one picture every $1 \mu \mathrm{m}$.

\section{Definition of photobleaching conditions}

To define the excitation parameters for photobleaching, and study the potential destructive effect of photobleaching, we made a test grid of photobleached squares with varying laser power and exposure time (figure 2). We computed total fluence, i.e. the total energy received per unit area for comparison. We started with low photobleaching at $53 \mathrm{~J} / \mathrm{mm}^{2}$ until very strong photobleaching at $2130 \mathrm{~J} / \mathrm{mm}^{2}$.

\section{Grid of photobleaching for strain field measurement}

In view of strain field measurement, a regular grid of photobleached squares was made in the field of view. This grid is composed of 16 squares (4x4) with a side of $20 \mu \mathrm{m}$ for each and a $40 \mu \mathrm{m}$ spacing, giving a total grid of $200 \mu \mathrm{m} \times 200 \mu \mathrm{m}$. Photobleaching was achieved according to the conditions selected previously: power was $50 \%$ of total power source - which is $600 \mathrm{~mW}$ at $850 \mathrm{~nm}$ for the laser considered; exposure time was set to $4 \mathrm{~s} / \mathrm{slice}$ and the scanning irradiation was repeated 3 times. Total fluence for this process was $90 \mathrm{~J} / \mathrm{mm}^{2}$, which was a reasonable choice resulting from a compromise between limited laser power/exposure time - to prevent laser damages to the sample - and good contrast for the photobleached squares. 
This process was performed in 2 distinct planes, spaced with approximately $20 \mu \mathrm{m}$, to allow strain measurement along the thickness direction. We did not quantify the change of total thickness of the samples because the upper and lower limits of our tissue are difficult to identify with great precision. Indeed, depending on the gain/offset settings, the identification of the first and last plane where we monitor a signal varies and therefore, we cannot get a good estimation of the sample total thickness. Following the position evolution of the two photobleached planes, we measured strain in the thickness direction. With such spacing between the two planes, we did not notice an impact of the depth of the focal plane on the intensity of photobleaching.

\section{Mechanical loading}

To perform uniaxial tensile test, we used an in situ micro tensile stage (DEBEN, UK). This stage is equipped with a $150 \mathrm{~N}$ load cell and a displacement transducer. As the two jaws are mobile, we chose a region of interest (ROI) close to the middle of the sample so that it did not move too much during loading to ensure imaging of the same ROI during testing. However, there was a limited displacement of the ROI which needed repositioning that we performed thanks to the photobleached grid. An immersion tray, as well as custom build jaws, were designed to perform these tests in an hydrated environment. Figure 1b) shows the experimental set up. Immersing sample answer several issues: it provides more realistic testing conditions, it ensures a water drop between the objective and the sample and last, it is much easier to handle liver's capsule in a wet state. Loading was conducted at 1 $\mathrm{mm} / \mathrm{min}$ speed. Samples were initially loaded to $0.05 \mathrm{~N}$ to ensure a flat initial state. Then, we performed a set of 5 loads/unloads between $0.05 \mathrm{~N}$ and $1 \mathrm{~N}$, to remain in the quasi linear section of the material behavior. We stopped around $15 \mathrm{~min}$ to image and photobleach our ROI, and performed another set of 5 identical cycles again. We repeated this loading pattern twice, changing ROI each time to investigate the photobleaching effect on mechanical properties in the quasi linear response of the material. Then, we conducted loading up to failure, stopping each $0.25 \mathrm{~N}$ to image one of our photobleached ROI. Figure 3 presents the loading path, where sections corresponding to image acquisition for microstructural observation are indicated. From our force-displacement measurements, we computed engineering stress and engineering strain, dividing force and displacement respectively by initial section and initial length.

\section{Analysis of the photobleached grids influence on macroscopic behavior}

To assess the effects of photobleaching at the macroscopic scale, sets of loads/unloads before and after photobleaching were compared. The mean and standard deviation of distances between curves were computed performing an orthogonal projection of the compared curve on the reference one.

On one hand, within a set of 5 loads/unloads, we compared each load/unload to the fifth one, to have a reference of acceptable curve distance measurement, assuming that the differences between loads/unloads within a same set of cycles are only due to normal re-organization of the structure.

On the other hand, we compared each load/unload of every set of cycles to the fifth one of the first set of cycles -the one before photobleaching. Considering the fifth load/unload of first set as reference allowed not to take into account all set up flaws like possible slippage or initial micro cracks, and to compare cycles when they become repeatable. Then, considering the measurements within a same set as reference for identical curves, we compared the measurements made between sets to gage at photobleaching effects.

\section{Strain field measurement}

Regarding local strain field measurement from the photobleached grid, positions of square's centers were collected manually via image segmentation using ImageJ. The good contrast between photobleached squares and the rest of image allows precise segmentation, down to 1 pixel accuracy. A Matlab script was developed to compute longitudinal, transverse and shear strains using the finite element method (FEM).

Using the coordinates of square's centers, a 4 nodes element mesh of 9 elements was made as seen on figure 4a). For each slice, longitudinal and transverse node displacements - $u, v$ respectively - were calculated forming a vector $\mathrm{U}$.

Based on bi-linear interpolation functions, the displacement gradient was calculated from the derivative of these functions presented here:

$$
\left[\begin{array}{l}
N_{1, x} \\
N_{1, y}
\end{array}\right]=\frac{1}{2 A}\left[\begin{array}{l}
-a+t * a \\
-a+s * a
\end{array}\right],\left[\begin{array}{l}
N_{2, x} \\
N_{2, y}
\end{array}\right]=\frac{1}{2 A}\left[\begin{array}{c}
a-t * a \\
-a-s * a
\end{array}\right],\left[\begin{array}{l}
N_{3, x} \\
N_{3, y}
\end{array}\right]=\frac{1}{2 A}\left[\begin{array}{l}
a+t * a \\
a+s * a
\end{array}\right],\left[\begin{array}{l}
N_{4, x} \\
N_{4, y}
\end{array}\right]=\frac{1}{2 A}\left[\begin{array}{c}
-a-t * a \\
a-s * a
\end{array}\right]
$$


Where $N_{k, x}$ is the derivative of the interpolation function with respect to x (longitudinal direction) and $N_{k, y}$ with respect to y (transverse direction); $s$ and $t$ are the local coordinates, $a$ and $A$ the side and area of reference square, as shown on figure $4 b$ ).

We calculated this displacement gradient at each 4 Gauss points defined by $[s, t]=[ \pm 0.5774 \pm 0.5774]$.

From this gradient, we obtain the Green-Lagrange strain tensor by

$$
E=\frac{1}{2}\left(\overrightarrow{\operatorname{grad}}(\vec{u})+\overrightarrow{\operatorname{grad}}(\vec{u})^{T}+\overrightarrow{\operatorname{grad}}(\vec{u}) \cdot \overrightarrow{\operatorname{grad}}(\vec{u})^{T}\right)
$$

Eventually, we computed four strain tensors per squares, resulting in 36 strain tensors per slice. Considering that the $\mathrm{x} y$ coordinates of photobleached squares do not change much from one slice to another, we computed mean value between the two photobleached planes segmented for strain tensors in each square and used a Matlab routine for color representation.

This new method of strain measurement is an alternative to digital image correlation which is usually used for large strain distribution analysis [42]-[44].

We computed Green-Lagrange strain in the thickness direction, based on the measurement of the distance between our two photobleached planes at each loading step, to get a first estimation of local strain in that direction.

\section{Results}

\section{Definition of photobleaching conditions}

Figure 2 shows the test grid on one slice as it appears in the fluorescence channel with indicated fluence for each square. There is not any apparent damage of the sample after the completion of the test grid with variable fluence. As photobleaching affects fluorescence but not SHG signal, we can still monitor the collagen fibers after photobleaching via the SGH channel. For fluence under $890 \mathrm{~J} / \mathrm{mm}^{2}$, SHG signal is still well present in the photobleached squares. It starts to disappear - which could correspond to a burnt hole - above this value but not on every slice which indicates that we have not reach the full destruction of the tissue even with the highest value of fluence tested.

To obtain clear markers by photobleaching and to avoid any damage of the tissue, we choose parameters to get a fluence rate of $90 \mathrm{~J} / \mathrm{mm}^{2}$ to perform the grid. Figure 5 shows the photobleached grid before (a) loading. With these conditions, we zoomed on one photobleached square to assess qualitatively the effects of photobleaching on the geometry of the fibers' network and to detect a potential burnt area. Figure 6 shows both SHG (a) and fluorescence (b) channel of a zoom on a photobleached square. Image analysis reveals that we still monitor a SHG signal in the square, showing that the collagen fibers have not suffered a priori from photobleaching and appear, structurally speaking, intact. The geometry of elastin fibers network does not appear modified either by this process, considering that even if we lost some fluorescence, there is still some signal left that allows us to monitor parts of the fibers which appears to have kept their continuity as seen on figure 6b). Besides, elastin fibers present around the square are not modified. We also repeated that zoom operation several times after loading to make sure that photobleaching does not modify the fibers' kinematic, observing qualitatively the behavior of collagen and elastin fibers. Elastin fibers extremities, as seen on the borders of the photobleached squares, seemed to have coherent kinematic with one another. Regarding collagen fibers, their behaviors were not different from what was previously observed.

\section{Comparison of macroscopic mechanical behaviors after photobleaching}

Figure 7 presents an example of the first set of 5 cycles (a) and a set of 5 cycles after 3 photobleachings (b). The 5 cycles within a set show a high repeatability, as well as loads/unloads between different sets. Table 1 shows mean values of inter curve distances and the associated standard deviations. The 'reference' line refers to calculation of mean distance values between loads/unloads of a same set. Averaging the results among the different samples, we find a mean distance of $4.0 \mathrm{kPa}(\mathrm{SD} 0.7$ ) and standard deviation of 3.0kPa (SD 5) that we consider as reference value for quasi identical curves. 
Except for the very first cycle of the first set - which take into account initial setting flaws and irreversible phenomenon like a first fiber alignment in the loading direction, cycles are very much alike from one another as shown by the low values of both mean and standard deviation of curve distances with respect to reference values.

\section{Local strain field measurement}

Figure 8 shows an example of strain field evolution during loading. Transverse strain far exceeds longitudinal strain in absolute value with a maximum transverse strain at $-0.311(\mathrm{SD} 0.066, \mathrm{n}=4$ ), while maximum longitudinal strain is 0.216 (SD $0.061, n=4)$. For shear strain, a great variability among the samples is observed. Figure 10 presents the compared evolution of global engineering strain with longitudinal local strain. We notice an identical trend for these two parameters but there is a random shift, more or less important depending on sample, between the two curves. The differences between global and local strain varies from $1.1 \%$ to $40 \%$, indicating that local strain might be very representative of global strain in some location or not at all in others.

Regarding computed strain in the thickness direction $-E_{\mathrm{zz}}-$, results were not satisfying. Figure 11 shows $E_{\mathrm{zz}}$ evolution with loading for the four samples tested, and illustrates the high uncertainty of the measurements. To compute accurately this strain, we need to identify two distinguishable planes that we can spot on image stacks at each loading step. Unfortunately, our localized photobleaching does not mark one plan but a stack of approximately $6 \mu \mathrm{m}$, so we cannot rely on that to identify the planes. Therefore, we spot some particular structures that we can easily distinguish to ensure that we consider the same plans each time. However, considering that along thickness, we have one image every $1 \mu \mathrm{m}$, we cannot reach a great accuracy on the position of our considered planes. Thus, we do not observe clear trend about $E_{\text {zz }}$ variations, and the low accuracy of our measurements hides a probable slight decrease that we observe during live imaging.

\section{Discussion}

The levels of fluence mentioned in this study are way above the values given in the literature for photobleaching experiments on collagen and elastin compounds reported in Marcu et al. [33]. This difference is due to the fact that usually, in studies about photobleaching, one focuses on the first appearance of this phenomenon to detect and avoid it, whereas in our case we need a complete photobleaching of our squares for good contrast and easy segmentation. Moreover, the laser type impacts greatly fluence levels and TPEF is more energetic and localized than classic confocal excitation [31], [41]. Eventually, fluence levels depend also on the wavelength and material considered [33].

Even with the high power laser values considered, we did not observe damage of the liver's capsule. The presence of SHG signal in the photobleach squares indicates that collagen fibers are still well present in the photobleaching area. We can also assume that elastin fibers were not destroyed as well, even if they are not visible anymore, considering that fibers on the very border of photobleached squares have not changed and do not seem burnt, cut or damaged. However, in some cases, we could see lightly the photobleached square on the SHG channel because of the not sufficient selectiveness of our filters. Indeed, the band pass of our SHG filter is too wide, and therefore, we collect a little bit of autofluorescence in this channel, just enough so that the photobleached square become visible. Anyway, it appears that photobleaching does not affect the tissue integrity as we did not notice once a beginning of rupture in any photobleached zone, which could have been the case if the laser had created burnt holes in the tissue. Moreover, qualitative observation of the zoom on photobleached squares reveals that the geometry of collagen fibers is not modified. We can reasonably assume that it is also the case for elastin fibers geometry considering that fibers around squares are not affected and the fibers seem to have kept their continuity.

The comparison of cyclic tests reveals that global behavior is not modified by photobleaching, as cycles do not change much after each photobleaching phase. The small discrepancy in cycle's shape is due to setting adjustments and vanishes after some cycles. Therefore, we do not observe any impact of photobleaching on global mechanical properties. This was nearly expected considering the small volume affected by this phenomenon.

If we consider the effect of photobleaching at the local scale, mechanical damage of the tissue would also appear on a local strain map analysis. Indeed, damage would induce systematic differences on strain within the grid between strain on the border and strain at the center. To evaluate possible trends on strain, we compute a norm of strain $\left(\|E\|=\left(E_{i j} E_{i j}\right)^{1 / 2}\right)$ which is presented on figure $\left.8 \mathrm{~d}\right)$ for sample $\mathrm{n}^{\circ} 3$. No particular trend was seen and 
especially, we do not notice a higher strain on the border of the grid which would have been the case if the tissue had suffered mechanical damage.

In regards to local strain field measurements, we observed on the 4 tested samples that transverse strain is greater than longitudinal strain in absolute value as shown in figures 8 and 9. This shows that structural effects might be predominant in the elongation process of the liver's capsule. It concurs with previous findings about tendons elongation mechanism, which is not entirely due to fiber elongation [6], [9], [10]. Screen et al. [6] highlighted the fact that tendons elongation is primarily due to fibers sliding, at different scales, while fibers elongation account for a small part of global extension. Fibers reorientation and uncrimping is also a key factor in tendon extension [10]. It is likely that we find the same mechanisms at work with Liver's capsule which, unlike tendons where fibers are already more or less pre-aligned in the tendon axis, is way less organized, and present a 'chaotic' network which induce many structural effects that are difficult to predict.

Therefore, those structural effects induce local changes of volume. Locally, the particular arrangement of fibers before stretching is very less organized. During loading, fibers align in one direction, and this reorganization induces an increase of fiber density in the considered volume. In fact the volume can be slightly reduced because the spacing between fibers is reduced with reorganization due to structural effects. Previous studies [45], [46] have reported the phenomenon of exudation of fluid from the extracellular matrix during loading of collagen fibers bundles, resulting in a loss of fluids of the structure that contributes to the reduction of interfibrillar space.

Moreover, shear strain values are quite heterogeneous among the samples. Depending on location, the fibers inside the ROI experience sliding one way or the other. Much like tendons [6], collagen fibers tend to slide from one to another where the link between them is the weakest. That explains why shear strain reaches important positive or negative values, when the sliding occurs in the photobleached zone, or why in some cases, shear strain barely differs from 0 , when the link between collagen fibers is strong enough to prevent sliding.

As expected, local and global longitudinal strains do not match perfectly, as seen on figure 10.However, given the sample's dog bone geometry, local strain should be higher than global strain which is a trend that we do not observe on figure 10. Indeed, depending on the location of the ROI, fibers experience different elongation/sliding intensities resulting in an heterogeneous local longitudinal strain throughout the sample which therefore, does not match an homogeneous behavior.

For strain along thickness measurements, we cannot draw any conclusions considering the high uncertainty when we followed the two reference planes which are photobleached. However, this method remains valid and would only need a smaller z-step during image acquisition. With this greater image density in the thickness direction, we could expect to reach a greater resolution down to the optical limit of the microscope. The main issue would be that a smaller z-step would lead to longer pauses during loading, with longer relaxation time and an important decrease of force measurement.

\section{Conclusion}

In this study, we present a new method to measure local strain field in connective fibrous membranes. We demonstrated the validity of using photobleaching to create markers on this tissue, showing that it does not impact macroscopic mechanical properties or collagen fibers' geometry, if performed at a certain fluence level. This technique is quit useful to study fiber based structures which have autofluorescent properties, which is the case for many biological tissues based on collagen-elastin fibers such as skin, arterial walls, tendons or other connective membranes. It allows positioning intrinsic markers on those tissues which can be used to various aims such as following a precise ROI during loading or measuring local strain in 3 dimensions as it is presented in this study. To have an insight on local strain field on such structures may allow understanding the contribution of microstructure to the macroscopic mechanical behavior of the tissue and to the damage and failure mechanisms. Such information are the starting point to develop structurally based models of materials behavior and can be used to help designing or validate the behavior of engineered biomaterials.

\section{Acknowledgments}

The authors thank the IVTV (ANR-10-EQPX-06-01) team for its help during the imaging process. 
This work was supported by the Programme Avenir Lyon Saint-Etienne (ANR-11-IDEX-0007) of Université de Lyon, within the program "Investissements d'Avenir" operated by the French National Research Agency (ANR).

\section{Disclosures}

None of the authors have any professional or financial conflict of interest.

\section{References}

[1] A. Brunon, K. Bruyère-Garnier, and M. Coret, "Characterization of the nonlinear behaviour and the failure of human liver capsule through inflation tests," J. Mech. Behav. Biomed. Mater., vol. 4, no. 8, pp. 1572-81, Nov. 2011.

[2] S. Umale, C. Deck, N. Bourdet, P. Dhumane, L. Soler, J. Marescaux, and R. Willinger, "Experimental mechanical characterization of abdominal organs: liver, kidney \& spleen.," J. Mech. Behav. Biomed. Mater., vol. 17, pp. 22-33, Jan. 2013.

[3] M. Perrini, W. Bürzle, C. Haller, N. Ochsenbein-Kölble, J. Deprest, R. Zimmermann, E. Mazza, and M. Ehrbar, "Contractions, a risk for premature rupture of fetal membranes: a new protocol with cyclic biaxial tension.," Med. Eng. Phys., vol. 35, no. 6, pp. 846-51, Jun. 2013.

[4] A. Ní Annaidh, K. Bruyère, M. Destrade, M. D. Gilchrist, and M. Otténio, "Characterization of the anisotropic mechanical properties of excised human skin.," J. Mech. Behav. Biomed. Mater., vol. 5, no. 1, pp. 139-48, Jan. 2012.

[5] B. D. Stemper, N. Yoganandan, and F. a Pintar, "Methodology to study intimal failure mechanics in human internal carotid arteries.," J. Biomech., vol. 38, no. 12, pp. 2491-6, Dec. 2005.

[6] H. R. C. Screen, D. A. Lee, D. L. Bader, and J. C. Shelton, "An investigation into the effects of the hierarchical structure of tendon fascicles on micromechanical properties.," Proc. Inst. Mech. Eng. H., vol. 218, no. 2, pp. 109-19, Jan. 2004.

[7] H. R. C. Screen, "Investigating load relaxation mechanics in tendon.," J. Mech. Behav. Biomed. Mater., vol. 1, no. 1, pp. 51-58, Jan. 2008.

[8] H. R. C. Screen and S. L. Evans, "Measuring Strain Distributions in Tendon using Confocal Microscopy and Finite Elements," J. Strain Anal. Eng. Des., vol. 44, no. 5, pp. 327-335, Jul. 2009.

[9] N. Sasaki and S. Odajima, "Force--Strain Mechanism of Collagen Fibrils and Relations of Tendon At Each Level of Structural," Science (80-. )., vol. 29, no. 9, pp. 1131-1136, 1995.

[10] Y. G. Houssen, I. Gusachenko, M.-C. Schanne-Klein, and J.-M. Allain, "Monitoring micrometer-scale collagen organization in rat-tail tendon upon mechanical strain using second harmonic microscopy.," $J$. Biomech., vol. 44, no. 11, pp. 2047-2052, Jul. 2011.

[11] J. T. Keyes, D. R. Lockwood, B. R. Simon, and J. P. Vande Geest, "Deformationally dependent fluid transport properties of porcine coronary arteries based on location in the coronary vasculature.," J. Mech. Behav. Biomed. Mater., vol. 17, pp. 296-306, Jan. 2013.

[12] J. T. Keyes, D. G. Haskett, U. Utzinger, M. Azhar, and J. P. Vande Geest, "Adaptation of a planar microbiaxial optomechanical device for the tubular biaxial microstructural and macroscopic characterization of small vascular tissues.," J. Biomech. Eng., vol. 133, no. 7, p. 075001, Jul. 2011. 
[13] J. Liao, L. Yang, J. Grashow, and M. S. Sacks, "Molecular orientation of collagen in intact planar connective tissues under biaxial stretch.," Acta Biomater., vol. 1, no. 1, pp. 45-54, Jan. 2005.

[14] P. P. Purslow, T. J. Wess, and D. W. Hukins, "Collagen orientation and molecular spacing during creep and stress-relaxation in soft connective tissues.," J. Exp. Biol., vol. 201, no. Pt 1, pp. 135-142, 1998.

[15] S. P. Lake, K. S. Miller, D. M. Elliott, and L. J. Soslowsky, "Effect of fiber distribution and realignment on the nonlinear and inhomogeneous mechanical properties of human supraspinatus tendon under longitudinal tensile loading.," J. Orthop. Res., vol. 27, no. 12, pp. 1596-602, Dec. 2009.

[16] S. P. Lake, K. S. Miller, D. M. Elliott, and L. J. Soslowsky, "Tensile properties and fiber alignment of human supraspinatus tendon in the transverse direction demonstrate inhomogeneity, nonlinearity, and regional isotropy.," J. Biomech., vol. 43, no. 4, pp. 727-732, Mar. 2010.

[17] A. M. Briones, J. M. González, B. Somoza, J. Giraldo, C. J. Daly, E. Vila, M. Carmen González, J. C. McGrath, and S. M. Arribas, "Role of elastin in spontaneously hypertensive rat small mesenteric artery remodelling.," J. Physiol., vol. 552, no. Pt 1, pp. 185-195, Oct. 2003.

[18] S. Boumaza, S. M. Arribas, M. Osborne-Pellegrin, J. C. McGrath, S. Laurent, P. Lacolley, and P. Challande, "Fenestrations of the carotid internal elastic lamina and structural adaptation in stroke-prone spontaneously hypertensive rats.," Hypertension, vol. 21, no. 1, pp. 1101-1107, Apr. 2001.

[19] C. B. Raub, J. Unruh, V. Suresh, T. Krasieva, T. Lindmo, E. Gratton, B. J. Tromberg, and S. C. George, "Image Correlation Spectroscopy of Multiphoton Images Correlates with Collagen Mechanical Properties," Biophys. J., vol. 94, no. 6, pp. 2361-2373, Mar. 2008.

[20] M. P. Rubbens, A. Driessen-Mol, R. a Boerboom, M. M. J. Koppert, H. C. Van Assen, B. M. TerHaar Romeny, F. P. T. Baaijens, and C. V. C. Bouten, "Quantification of the Temporal Evolution of Collagen Orientation in Mechanically Conditioned Engineered Cardiovascular Tissues," Ann. Biomed. Eng., vol. 37, no. 7, pp. 1263-1272, Jul. 2009.

[21] A. Zoumi, A. Yeh, and B. J. Tromberg, "Imaging cells and extracellular matrix in vivo by using secondharmonic generation and two-photon excited fluorescence.," Proc. Natl. Acad. Sci. U. S. A., vol. 99, no. 17, pp. 11014-9, Aug. 2002.

[22] A. Zoumi, X. Lu, G. S. Kassab, and B. J. Tromberg, "Imaging coronary artery microstructure using second-harmonic and two-photon fluorescence microscopy.," Biophys. J., vol. 87, no. 4, pp. 2778-2786, Oct. 2004.

[23] Z. Deyl, K. Macek, M. Adam, and O. Vancíková, "Studies on the chemical nature of elastin fluorescence.," Biochim. Biophys. Acta, vol. 625, no. 2, pp. 248-54, Oct. 1980.

[24] E. Fujimori, "Changes induced by ozone and ultraviolet light in type I collagen. Bovine Achilles tendon collagen versus rat tail tendon collagen.," Eur. J. Biochem., vol. 152, no. 2, pp. 299-306, Oct. 1985.

[25] D. Fujimoto, K. Akiba, and N. Nakamura, "Isolation and characterization of a fluorescent material in bovine achilles tendon collagen," Biochem. Biophys. Res. Commun., vol. 76, no. 4, pp. 1124-1129, Jun. 1977.

[26] R. Richards-Kortum and E. Sevick-Muraca, "Quantitative optical spectroscopy for tissue diagnosis.," Annu. Rev. Phys. Chem., vol. 47, pp. 555-606, Jan. 1996.

[27] P. J. Campagnola, A. C. Millard, M. Terasaki, P. E. Hoppe, C. J. Malone, and W. A. Mohler, "Threedimensional high-resolution second-harmonic generation imaging of endogenous structural proteins in biological tissues.," Biophys. J., vol. 82, no. 1 Pt 1, pp. 493-508, Jan. 2002. 
[29] E. H. K. Stelzer, "Contrast, resolution, pixelation, dynamic range and signal- to-noise ratio : fundamental limits to resolution in fluorescence light microscopy," vol. 189, no. January, pp. 15-24, 1998.

[30] T. Bernas, M. Zarebski, R. R. Cook, J. W. Dobrucki, and P. R. Cook, "Minimizing photobleaching during confocal microscopy of fluorescent probes bound to chromatin: role of anoxia and photon flux.," J. Microsc., vol. 215, no. Pt 3, pp. 281-96, Sep. 2004.

[31] A. Diaspro, G. Chirico, C. Usai, P. Ramoino, and J. Dobrucki, "Photobleaching," in in Handbook Of Biological Confocal Microscopy SE - 39, J. B. Pawley, Ed. Springer US, 2006, pp. 690-702.

[32] C. Kuang, D. Luo, X. Liu, and G. Wang, "Study on factors enhancing photobleaching effect of fluorescent dye," Measurement, vol. 46, no. 4, pp. 1393-1398, May 2013.

[33] L. Marcu, W. S. Grundfest, and J. M. Maarek, "Photobleaching of arterial fluorescent compounds: characterization of elastin, collagen and cholesterol time-resolved spectra during prolonged ultraviolet irradiation.," Photochem. Photobiol., vol. 69, no. 6, pp. 713-21, Jun. 1999.

[34] L. Song, E. J. Hennink, I. T. Young, and H. J. Tanke, "Photobleaching kinetics of fluorescein in quantitative fluorescence microscopy.," Biophys. J., vol. 68, no. 6, pp. 2588-600, Jun. 1995.

[35] N. B. Vicente, J. E. D. Zamboni, J. F. Adur, E. V Paravani, and V. H. Casco, "Photobleaching correction in fluorescence microscopy images," J. Phys. Conf. Ser., vol. 90, p. 012068, Nov. 2007.

[36] X. Zhang, Q. Li, J. Liu, and S. Sottini, "Chemical mechanism of photobleaching of poly-3BCMU film," vol. 95, pp. 239-244, 1996.

[37] D. Axelrod, D. E. Koppel, J. Schlessinger, E. Elson, and W. W. Webb, "Mobility measurement by analysis of fluorescence photobleaching recovery kinetics.," Biophys. J., vol. 16, no. 9, pp. 1055-69, Sep. 1976.

[38] H. C. Ishikawa-Ankerhold, R. Ankerhold, and G. P. C. Drummen, "Advanced fluorescence microscopy techniques--FRAP, FLIP, FLAP, FRET and FLIM.,” Molecules, vol. 17, no. 4, pp. 4047-132, Jan. 2012.

[39] G. a Dunn, I. M. Dobbie, J. Monypenny, M. R. Holt, and D. Zicha, "Fluorescence localization after photobleaching (FLAP): a new method for studying protein dynamics in living cells.," J. Microsc., vol. 205, no. Pt 1, pp. 109-12, Jan. 2002.

[40] V. W. T. Cheng and H. R. C. Screen, "The micro-structural strain response of tendon," J. Mater. Sci., vol. 42, no. 21, pp. 8957-8965, Jul. 2007.

[41] G. H. Patterson and D. W. Piston, "Photobleaching in two-photon excitation microscopy.," Biophys. J., vol. 78, no. 4, pp. 2159-62, Apr. 2000.

[42] C. Sachs, H. Fabritius, and D. Raabe, "Experimental investigation of the elastic-plastic deformation of mineralized lobster cuticle by digital image correlation.," J. Struct. Biol., vol. 155, no. 3, pp. 409-25, Sep. 2006.

[43] D. Zhang, C. D. Eggleton, and D. D. Arola, "Evaluating the Mechanical Behavior of Arterial Tissue using Digital Image Correlation,” no. Dic, pp. 409-416, 2002.

[44] A. Brunon, K. Bruyère-Garnier, and M. Coret, "Mechanical characterization of liver capsule through uniaxial quasi-static tensile tests until failure.," J. Biomech., vol. 43, no. 11, pp. 2221-2227, Aug. 2010.

[45] Y. Lanir, E. L. Salant, and A. Foux, "Physico-chemical and microstructural changes in collagen fiber bundles following stretch in-vitro.," Biorheology, vol. 25, no. 4, pp. 591-603, Jan. 1988. 
[46] J. A. Hannafin and S. P. Arnoczky, "Effect of cyclic and static tensile loading on water content and solute diffusion in canine flexor tendons: an in vitro study.," J. Orthop. Res., vol. 12, no. 3, pp. 350-6, May 1994. 


\begin{tabular}{|c|c|c|}
\hline & \multicolumn{2}{|c|}{ Inter curve distances $(\mathrm{kPa})$} \\
\hline & $\begin{array}{c}\text { Average } \\
(\text { npoints } \approx 600, \text { ncycles }=5)\end{array}$ & $\begin{array}{c}\text { Standard deviation } \\
\text { (npoints } \approx 600, \text { ncycles }=5 \text { ) }\end{array}$ \\
\hline Reference & $4.0($ SD 0.7) $(n=4)$ & $3.0($ SD 0.5) $(n=4)$ \\
\hline No photobleaching & $5.1(\mathrm{SD} 0.7)(\mathrm{n}=4)$ & $3.5(\mathrm{SD} 0.7)(\mathrm{n}=4)$ \\
\hline 1 grid of photobleaching & $4.3(\mathrm{SD} 0.5)(\mathrm{n}=4)$ & $2.7($ SD 0.1) $(n=4)$ \\
\hline 2 grids of photobleaching & $4.2(\mathrm{SD} 0.4)(\mathrm{n}=4)$ & $2.7(\mathrm{SD} 0.1)(\mathrm{n}=4)$ \\
\hline 3 grids of photobleaching & $4.8(\mathrm{SD} 1.4)(\mathrm{n}=4)$ & $2.8(\mathrm{SD} 0.2)(\mathrm{n}=4)$ \\
\hline
\end{tabular}

Table 1: Average values and standard deviation of inter curve distances. Reference refers to distances within a same set of 5 cycles which are assumed to represent typical distances between very similar curves. Values are given with standard deviation with respect to inter samples variability $(n=4)$. Average and standard deviation of inter curve distances are mean values calculated over approximately 600 points and averaged on the five cycles within a same set.

\section{Figure legends}

Figure 1: a) Description of samples' geometry with positions of photobleaching grids. b) Experimental set up. The custom build immersion tray is put on the in situ micro tensile stage (DEBEN, UK). Immersion tray is in two parts to follow jaws' displacements. Waterproofness is achieved by plastic wrap

Figure 2: Photobleached test grid as it appears on the fluorescence channel. Horizontally are different laser powers from $3 \%$ to $30 \%$ of total power source $(600 \mathrm{~mW})$. Vertically are different exposure times varying from $2 \mathrm{~s}$ to $8 \mathrm{~s}$. Test squares dimensions are $26 \mu \mathrm{m} \times 26 \mu \mathrm{m}$. Total fluence per photobleached square is computed (as Power $x$ Exposure time / square area) and shown in yellow.

Figure 3: Loading path (sample $\mathrm{n}^{\circ} 4$ ). It begins with 4 cycles of 5 loads/unloads between $0.05 \mathrm{~N}$ and $1 \mathrm{~N}$. There are $\approx 15 \mathrm{~min}$ pauses between sets of cycles to perform photobleaching. Then sample is loaded up to failure with pauses every $0.25 \mathrm{~N}$ for image acquisition of the photobleaching grid. Red sections correspond to image acquisition.

Figure 4: a) 4 nodes element mesh built from the photobleaching grid in a deformed state. The centers of photobleached squares (in red) represent the nodes of the mesh which is composed of 9 elements. In blue are the Gauss/integration points where the gradient of displacement is calculated. Therefore we compute 4 GreenLagrange strain tensors per element and use a Matlab routine to color each quarter of the elements according to the associated value of strain. b) Definition of Gauss points/ integration points in the finite element mesh

Figure 5: Grey scale images of the human liver's capsule as seen on the fluorescent channel of the two-photon excitation microscope for sample $\mathrm{n}^{\circ}$ 4. a) Photobleaching grid before loading (force level $\mathrm{F}=0.07 \mathrm{~N}$ ). Initial squares' dimensions are $20 \mu \mathrm{m}$ x $20 \mu \mathrm{m}$ with $40 \mu \mathrm{m}$ spacing between them. b) Photobleaching grid after loading (force level $\mathrm{F}=1.76 \mathrm{~N}$ )

Figure 6: Zoom (x10) on a photobleached square before loading (force level $F=0.01 \mathrm{~N}$ ). Total field of view is $51.2 \mu \mathrm{m} \times 51.2 \mu \mathrm{m}$ with a resolution of $0.05 \mu \mathrm{m} /$ pixel. a) Second Harmonic Generation (SHG) channel showing the collagen fibers. b) Fluorescence channel showing mainly elastine fibers and the photobleached square.

Figure 7: a) First set of 5 cycles (sample $n^{\circ} 4$ ) of human liver's capsule loading as seen on the engineering stressstrain curve. Loading is conducted between $0.05 \mathrm{~N}$ and $1 \mathrm{~N}$ at $1 \mathrm{~mm} / \mathrm{min}$ speed. Every cycle of every set is compared to the fifth cycle of this set (shown in red). b) $4^{\text {th }}$ set of 5 cycles showing the repeatability of the liver capsule engineering stress-strain behavior (sample $\mathrm{n}^{\circ} 4$ )

Figure 8: Green-Lagrange strain field evolution during loading (sample $n^{\circ} 3$ ) showing the heterogeneity of local strain in human liver's capsule. Each image is associated to a level of loading indicated by the average force level measured during image acquisition. Each square represents the value of Green Lagrange strain calculated at a specific Gauss point.

a) Longitudinal strain 
b) Shear strain

c) Transverse strain

d) $\operatorname{Norm}\left(\|E\|=\left(E_{i j} E_{i j}\right)^{1 / 2}\right)$

Figure 9: Variation of mean longitudinal strain $\left(E_{x x}\right)$ with respect to mean transverse strain $\left(E_{y y}\right.$ shown in red) and mean shear strain ( $E_{x y}$ shown in black) for the 4 samples tested. While shear strain does not vary much, transverse strain becomes greater than longitudinal strain in absolute value.

Figure 10: Global/Local longitudinal strain comparison on the 4 samples. $E_{x x}$ local correspond to the average value of local longitudinal strain over the photobleached grid calculated at the different loading level of image acquisition.

Figure 11: Green Lagrange strain evolution in the thickness direction for the 4 samples tested (error bars represent the uncertainty of the measurement) at the different levels of loading corresponding to image acquisition. 